\title{
DOE Robotic and Remote Systems Assistance to the Government of Japan
}

Derek Wadsworth

Victor Walker

February 2013

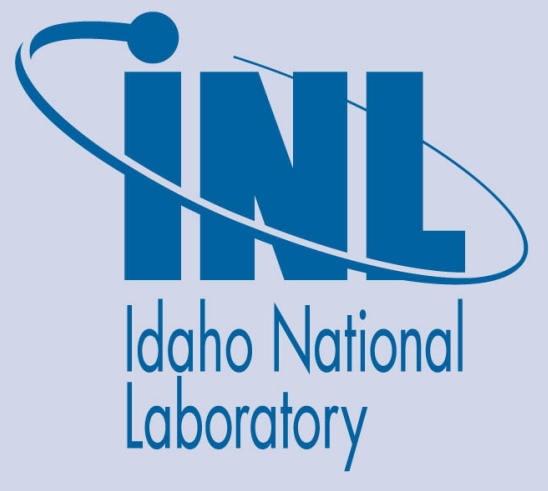

The INL is a U.S. Department of Energy National Laboratory operated by Battelle Energy Alliance 
INL/EXT-13-28233

\title{
DOE Robotic and Remote Systems Assistance to the Government of Japan
}

\author{
Derek Wadsworth \\ Victor Walker
}

February 2013

\begin{abstract}
Idaho National Laboratory
Idaho Falls, Idaho 83415
\end{abstract}

http://www.inl.gov

\author{
Prepared for the \\ U.S. Department of Energy \\ Office of Nuclear Energy \\ Under DOE Idaho Operations Office \\ Contract DE-AC07-05ID14517
}




\section{BACKGROUND}

On March 11, 2011 Japan experienced a series of disasters. After the terrible impacts of a major earthquake and tsunami, the resulting damage to the Fukushima Daichi nuclear plant threw much of the nation and world into turmoil.

In the days and weeks that followed there was a great need by the local responders and national decision-makers for information about plant damage and operational status as well as situation awareness with regard to radiation releases. The hazards represented by the radioactive environment made it hazardous for the staff to collect the necessary data. Automated recording devices were generally not functional or not relevant to the dynamic environment and there was need for more specific information. These conditions made it desirable to use remote systems technologies to gather relevant information. Within two weeks, the government of Japan and the owner of the nuclear plant contacted the United States to start an ongoing dialog of support and assistance in their time of need. The Idaho National Laboratory led a robotic response team including industry and several partners from other Department of Energy Laboratories. Those partnering laboratories included Sandia National Laboratory, Oak Ridge National Laboratory, Argonne National Laboratory, Pacific Northwest National Laboratory, and Savannah River National Laboratory. The mission given the team was to identify remote/robotic equipment available within the DOE inventory that could be made available to the Government of Japan (GoJ) to assist in assessing and recovering from the disaster.

An initial assessment of available inventory identified several radiation hardened camera systems, a number of radiation detectors, thermal imaging devices, highresolution cameras, and a collection of robotic vehicles for deploying measurement and inspection devices. This equipment list along with possible uses and means of deployment were presented to representatives of the GoJ, the Japanese Atomic Energy Agency (JAEA), and the Minister of Economy, Trade and Industry (METI). Following review and discussion with this group it was determined that the US DOE would make available to the GoJ equipment that could be used to assess the general condition and radiation levels in the vicinity surrounding the damaged reactors and surrounding facilities, and provide some feedback inside the structures. These assessments would be accomplished by appropriate sensors including cameras (still, video, radiation-hardened.), radiation detectors, and potential sample collection, etc. A key consideration in gathering the needed data was deployment of the sensors. It was determined that in areas where radiation levels were elevated that sensors would best be deployed by a remotely operated system or vehicle. The improved characterization of the situation was deemed to be critical, as it would dictate subsequent decisions and actions. In addition to the equipment the US DOE also offered, for consultation and support, technical experts with a wide range of expertise and experience developing and using robotic and remote systems and technologies, particularly in nuclear or radiological environments. 
The following table presents the equipment that was identified and delivered in response to the Japanese request.

Table I. Equipment made available to the GoJ.

\begin{tabular}{|l|c|c|c|}
\hline \multicolumn{1}{|c|}{$\begin{array}{c}\text { Item } \\
\text { Description }\end{array}$} & $\begin{array}{c}\text { NumberA } \\
\text { vailable }\end{array}$ & $\begin{array}{c}\text { Equipment } \\
\text { Location }\end{array}$ & $\begin{array}{c}\text { Replacement } \\
\text { Cost }\end{array}$ \\
\hline QinetiQ TALON & 1 & INL (BEA) & $\$ 250,000$ \\
\hline GammaCam & 1 & INL (CWI) & $\$ 60,000$ \\
\hline Radiation Detectors & 2 & INL (BEA) & $\$ 10,000$ \\
\hline Radiation Hardened Camera Systems & 2 & $\begin{array}{c}\text { 1-INL (BEA) } \\
\text { 1-ORNL }\end{array}$ & $\$ 30,000$ \\
\hline
\end{tabular}

\section{Preparation and Deployment}

Immediately following identification of the equipment to be sent, preparations were underway to provide not only the equipment but also the capabilities that would allow its use to accomplish the desired result. The Talon robot, manufactured by QinetiQ North America and owned by INL, was equipped with a radiation detector and a GPS unit. The intent was to have the robot perform radiation surveys outside the reactor and support buildings. Additionally, the Talon was equipped with extended radiation level detection capabilities and the capability to "paint" radiation levels on top of a Google earth map of the area. These capabilities were developed within a week after receiving word that the Talon would be shipped. These capabilities remain unique to the INL supplied robot and are shown in Figure 1. This image and associated data were collected at the Tsukuba City test sight where the DOE equipment was delivered and prepared by INL personnel for deployment. It is important to note that the radiation levels shown in the figure, represented by varying colors, are simulated and not actual readings. This was done for training purposes. The second picture represents readings later recorded by the Japanese at the sight and shared with INL. 


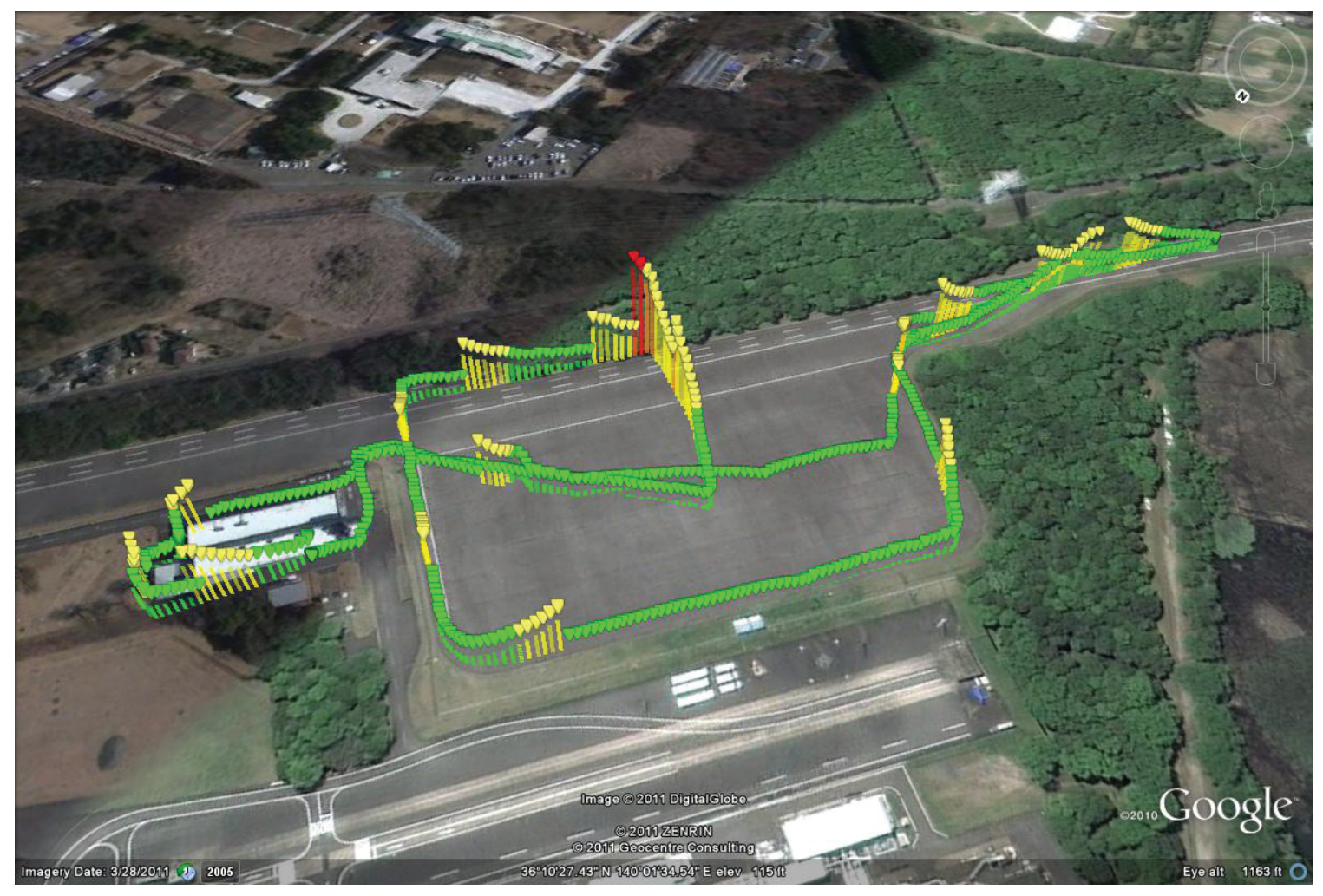

A. Training facility in Tsukuba City

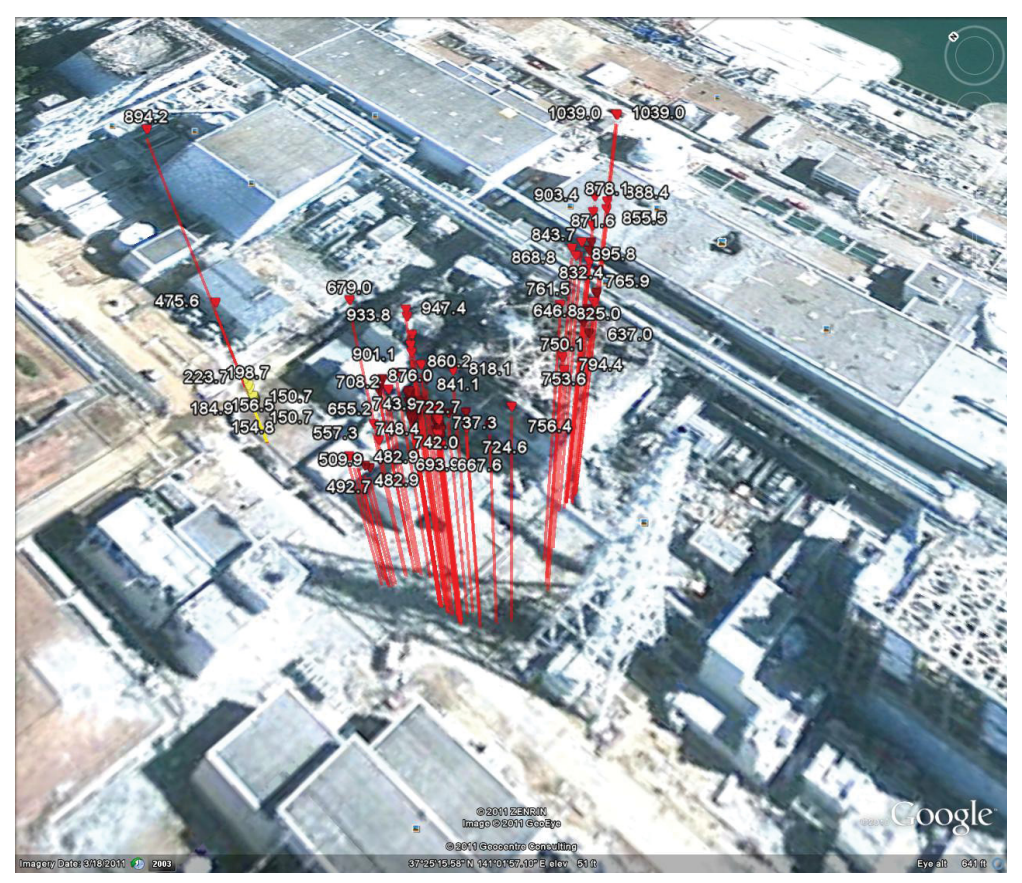

B. Later reports demonstrating use at the nuclear plant

Figure 1. INL Talon radiation mapping capabilities. 
Following Shipment of the equipment, a team of 4 engineers was sent to Tokyo Japan to set up the DOE equipment, provide training on the equipment, and to offer expertise in remote operations that could assist the Japanese in their recovery efforts of the Fukushima Daiichi nuclear reactor disaster.

After arriving in Japan, the team traveled to Tsukuba City, where a facility was made available for the INL team to setup the Talon Robot and mapping sensor, the GammaCam, and the radiation hardened cameras. At the initial meeting there were a host of individuals from the Japanese Government, TEPCO, JAEA and other organizations. The morning was spent with the Japanese presenting the intended use of the equipment, and the INL presenting the capabilities of the equipment that was sent. A "Ceremony" was conducted where the equipment was turned over by Ron Cherry (Energy Attaché Director, DOE Japan Office U.S. Embassy Tokyo), to Kentaro Funaki, (Director of Nuclear Policy for Ministry of Economy, Trade and Industry (METI)). Figure 2 shows the team of Japanese scientists and engineers that would use the equipment.

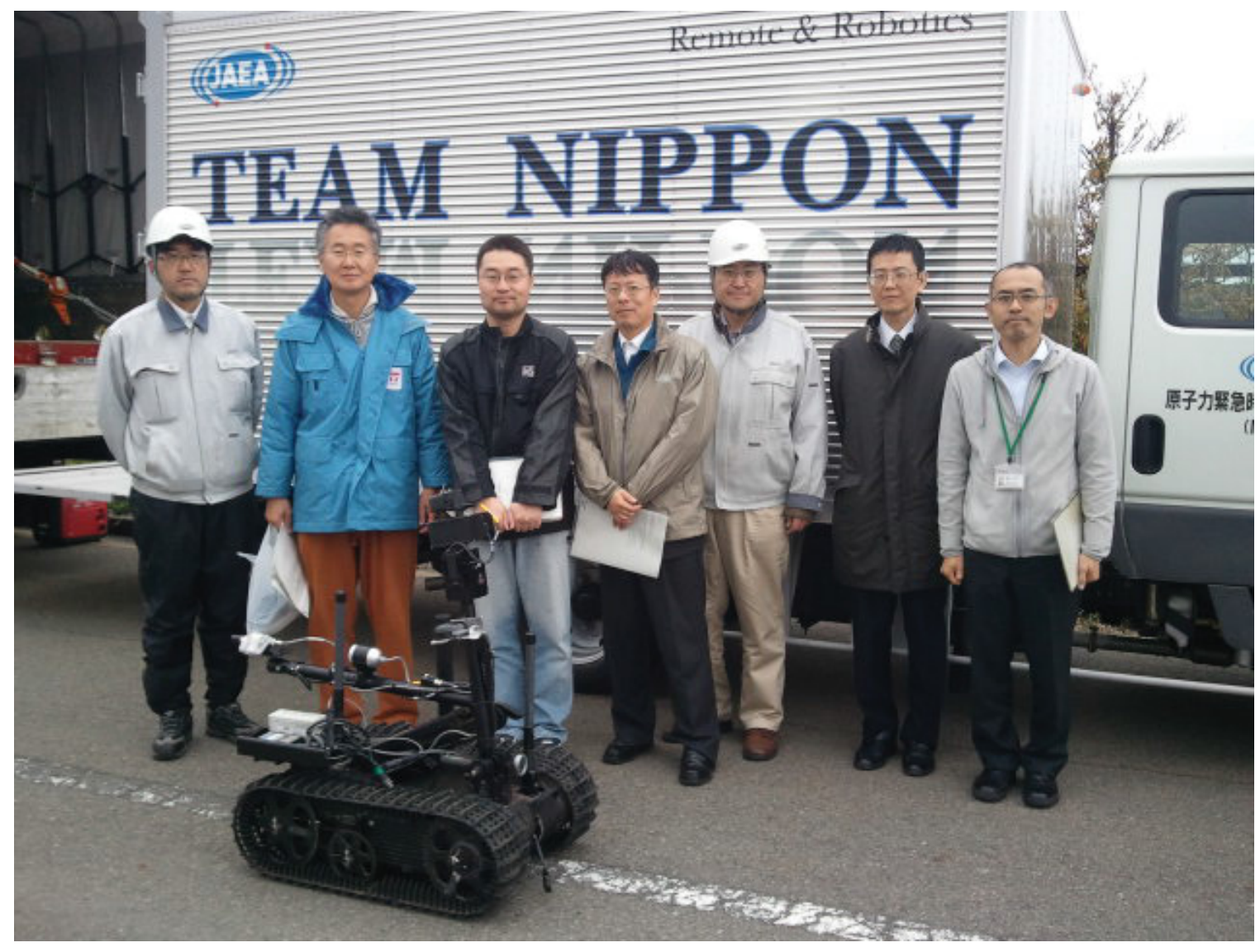

Figure 2. Japanese team. 
At this briefing it was presented by the TEPCO lead engineer (Dr. Shin Yoshino) that the equipment would be used as follows:

1 - Engineers from the Fukushima Daiichi nuclear reactor would identify "needs" to support a specific activity or issue.

2 - These needs were taken to a 6-member panel

3 - This panel would assign the need to one of its focus areas and determine/approve the use of existing equipment in addressing the need.

The panel identified that the immediate need for the DOE provided equipment was to map the radiation levels outside the buildings of the plant with the Talon robot thus avoiding an operator approaching some high-level areas while performing the task manually. They also elected to employ the GammaCam to measure and monitor radiation levels in areas around the plant - beginning with the delivery entrance to reactor 3 . This was a priority because they needed to understand and quantify the hazards associated with entry to the plant.

The INL team trained a team of TEPCO Engineers on the equipment. The assigned TEPCO engineers were very conscientious and thorough but it was obvious that they were not acquainted with the technologies provided and were not normally assigned to field deployment activities and would not be the final operators. The engineers from the INL and TEPCO struggled to communicate due to the language differences. No interpreter was provided. After two days of training, the equipment was taken to Hitachinaka where the GammaCam and Talon were prepared for an inspection and radiation mapping activity on the reactor 3 truck loading bay. The INL team traveled to Hitachinaka and supported the integration of the equipment into a shield box that JAEA developed to allow operators to get close to the areas of interest at reactor 3 . Figure 3 displays the INL equipment integrated into the Japanese provided "shield car".

The Japanese engineers traveled to the damaged reactor site to train the actual operators of the equipment. INL personnel were not allowed within the 15 mile radius restricted reactor buffer zone; and therefore, were unable to participate directly with the actual deployment of the robotic system. 


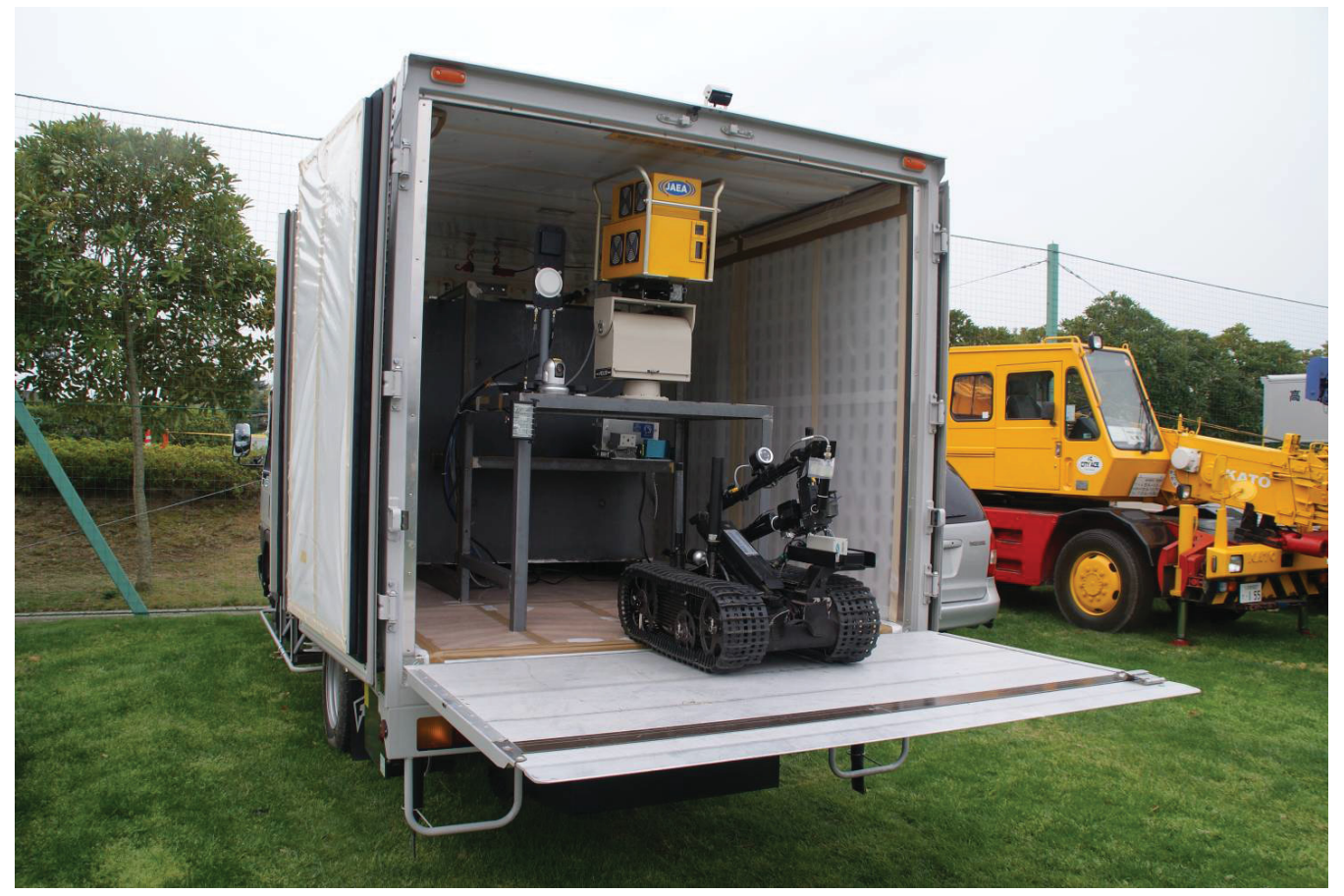

Figure 3. INL provided equipment ready for deployment.

INL personnel supported the Japanese operators for approximately two weeks in Japan. During that time they assisted in the deployment of equipment and trouble shooting of both INL provided equipment and equipment previously purchased by the Japanese Atomic Energy Agency. Following their return to the US, the INL personnel continued to offer support and consultation to the Japanese operators for the next three to four months.

\section{Equipment and Capability Replacement}

Upon return to the United States and following the initial deployment support, efforts were initiated to replace the equipment that was supplied to the GoJ. Funds were transferred to Oak Ridge National Laboratory to replace the camera system they provided. Funds were also provided to CWI to replace the Gamma Cam that they provided. Replacements for the INL radiation hardened camera systems were ordered and received.

The most difficult item to fully replace was the Talon robotic platform. The Talon platform sent to Japan was a modified version of QinetiQ North America's (QNA) stock Generation IV Talon. INL had teamed with QNA over a 4-year period in the development of the specialized Talon for use in autonomous hazard mapping. As such the platform supplied to the Japanese had a specialized navigational package that allowed for the integration of multiple sensors and installation of the INL Robotic Intelligence Kernel (RIK). The RIK is an operating system for robotic platforms. In a sense it does for robots what Microsoft Windows does for PCs. Just 
as MS Windows provides an interface environment and provides all the required protocols for ancillary devices, the RIK provides an operator user interface to a robotic platform and provides the necessary "hooks" into the robotic platform to allow the plug-and-play of multiple sensors. The RIK ensures that when a new sensor is plugged into the robot that the operating system recognizes the sensor, knows how to communicate with the sensor, and knows how to interpret the data returned from the sensor. The RIK also facilitates the locomotion of the robot allowing it to operate in a tele-operated mode (operator driving via joy stick) to a completely autonomous mode (sensors on the robotic platform provide obstacle location data and the robot drives itself).

Receipt of the replacement Talon was delayed initially because QinetiQ supplies the Talon to the armed services and we had to get in the queue. With tens of platform orders ahead of us it took several months to take delivery of the Talon. Once the Talon was received, it was discovered we had received a "generic" Talon that was not equipped with the necessary software to operate the specialized navigational package. The robot was returned to QNA and the proper software was installed. In the mean time, because the navigational module is a special order item, it had to be fabricated. This is not a high volume item for QNA and so it is only fabricated on request. To QNA's credit, they provided us with a loaner navigational package so when the robot was delivered we could initiate hardware integration and testing. The INL navigational package arrived in late November 2012. It was quickly installed on the new INL Talon at our facilities and tested for basic functionality. Loading of the RIK software onto the navigational package followed initial testing. Following software installation, debugging and testing ensued. Testing and debugging continued through December with final checkout of all functionality occurring the first week in January. As of now all capabilities and resources have been restored to all organizations supporting the Fukushima robotic and sensor response.

\section{Lessons Learned}

Key to employing national assets is a clear understanding of expectations and requirements also critical is the support of the agency that owns the assets. Both of these criteria were met during the Fukishima response. Clear communication and objectives were relayed and the DOE acted as a facilitator in accomplishing the equipment transfer. Communications with the government of Japan was difficult at times and resulted in some delays in using the equipment, which might have been avoided with clearer understanding of the requirements and culture of the organizations.

Critical to the continued capabilities of the national laboratories is replacement of resources if expended or transferred to other entities. The time to consider replacement is in the initial assessment of equipment to be employed. Some one-ofa-kind systems may be difficult to replace and require not only long procurement times but in addition, restoration of capability that has been added to the stock item. When the immediacy of the event wanes, attention should be immediately focused 
on restoration of capability. This will prevent gaps in the ability for the lab to respond to occurrences requiring the specialized capability.

The national laboratory system has a multitude of capabilities and resources that could be deployed in response to a national or international event; however, as evidenced by the impact of the natural disaster on the Fukushima nuclear reactor complex, there is a lack of prepositioned, performance validated equipment ready to provide a rapid, first-response assessment of a radiological incident (e.g., reactor failure, radiation release, etc.). The protocol and infrastructure to identify, evaluate, integrate, and maintain a suite of equipment to carry out and support a rapid assessment activity needs to be developed.

Upon evaluating previous nuclear incidents it is apparent that a critical need in the immediate aftermath of an event is a detailed assessment of the situation. This assessment involves collecting information at both wide area and localized sites involved in the incident. Parameters of interest may include imagery, radiation levels, temperature, and other environmental factors. These are key indicators for determining the extent of damage. Sensors to gather parameter data can be deployed using a variety of remotely operated vehicles, including fixed wing, rotorcraft, and ground based robots.
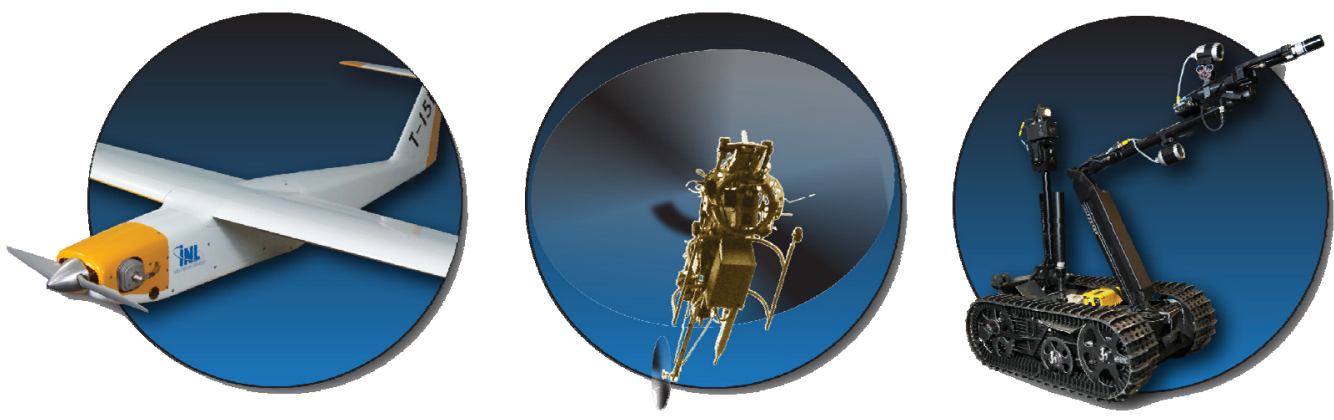

Sensor deployment platforms

A phased approach to the assessment is necessary with each phase collecting specific information and requiring special tools. Typical assessment phases are: (1) collection of environmental parameters, (2) determination of conditions driving environmental parameters, and (3) physical sample collection and analysis. The ideal solution would include utilization of commercially available technologies, which could be integrated into assessment systems. Additionally, specialized tools would be developed if standard systems were unable to provide required information. 
Phase I - First Look: Environmental parameter monitoring involves evaluating general conditions surrounding the reactors, facilities, and structures. These assessments can be accomplished using a variety of sensors including cameras (still, video, infrared, etc.), radiation detectors, and temperature sensors. A key component to gathering this data is the deployment of these sensors, which is typically accomplished by remotely operated ground or aerial vehicles. In order to gain a clear understanding of the situation, the data collected in this phase must then be characterized. This step is critical and should be the first priority of any assessment, as it will dictate subsequent decisions and actions.

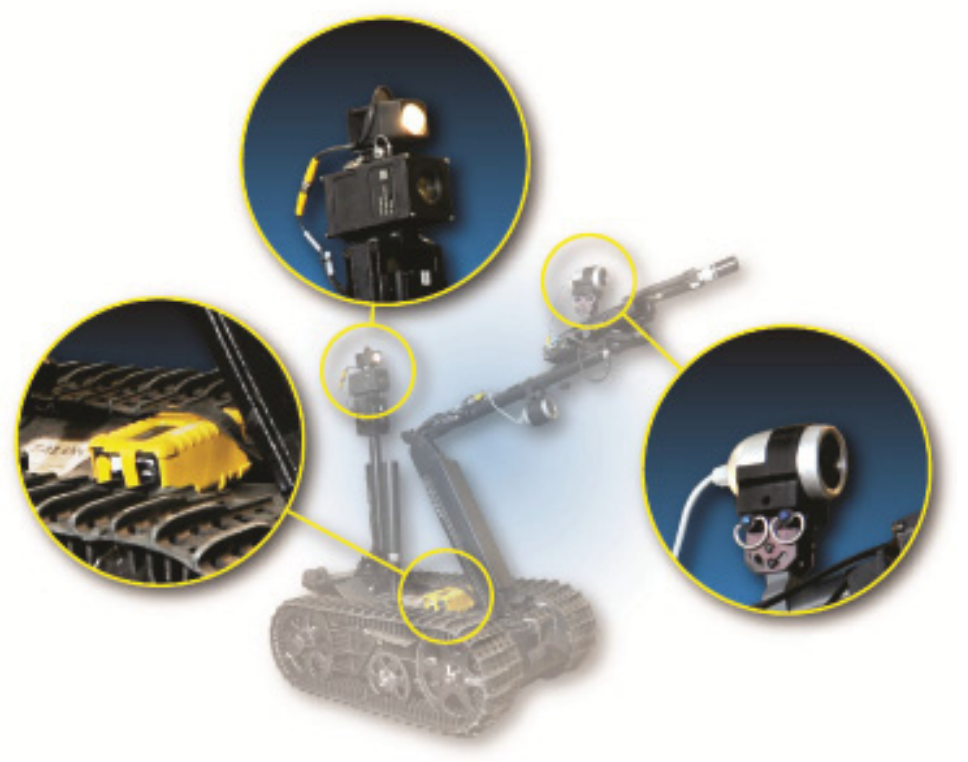

Robotic deployment platform with multiple sensors

Phase II - Situation Evaluation: Identifying the contributing conditions of the situation provides visual and other qualitative information that can be used to plan mitigating activities. This phase requires access to areas inside buildings and structures, which in turn requires wireless data/control transmission, platforms that can place sensors at key locations, non-line-of- sight operation, and other technology needs. During this phase, equipment is exposed to elevated radiation levels that may impact operation and longevity. Understanding these affects will assist in equipment selection, use, and maintenance.

Phase III - Sample Acquisition: Collecting physical samples allows response personnel to quantify the parameters that are present, leading to a greater understanding of how much damage has been sustained and what required recovery actions must be taken. The sampling activities-which include air and water sam- 
pling and potentially recovery of selected materials - are fairly unique and require general remote material handling capabilities.

Implementation of this strategy would result in Rapid Assessment Incident Deployment (RAID) Kits for each assessment phase. These kits would include information-gathering tools tailored to each phase of the assessment. These tools would be pre-screened using simulated accident environments (radiation levels, temperature ranges, and humidity levels) to assess their functionality under simulated accident conditions. This assessment would provide first responders with a set of guidelines on tool operating parameters including usable life. For example, the guidelines would allow first responders to know the expected lifetime of a digital camera as a function of radiation levels.

The tools included in the kits would be selected for a set of objectives defined for each phase of assessment. For example, the contents of the Phase I rapid response kit would provide a "first look" at on-the-ground factual information using simple, commonly available, inexpensive tools that could be discarded after exposure to the environmental conditions at the accident site. This would enable rapid acquisition of information without concern over the availability and the fate of expensive and potentially one-of-a-kind tools. The kits would be assembled and staged in appropriate shipping containers for rapid deployment by ground or air.

The government should consider the development of these kits through the Office of Nuclear Energy to provide DOE the ability to effectively support incidents involving nuclear power systems. INL is uniquely qualified to develop and test these kits for two reasons. First, INL has the test bed facilities and resources to conduct radiation and other severe environment testing of various sensors and equipment to determine the lifetime of off-the-shelf products such as cameras in extreme radiation environments. Second, INL has a robust program in place for developing tools and robotics for first responders. INL's legacy of nuclear power research on experimental and demonstration reactor systems built the capability for development of systems and tools to make difficult measurements in extreme environments. This capability coupled with INL's remote systems experience would provide the necessary expertise to establish and maintain a Rapid Assessment Incident Deployment Program for the Department of Energy. 